\title{
Surface ground improvement to reduce liquefaction damage to small structures
}

\author{
Hideyuki Mano ${ }^{\text {i) }}$, Yasuhiro Shamoto ${ }^{\text {ii) }}$ and Akira Ishikawa ${ }^{\text {iii) }}$
}

i) Group manager, Institute of Technology, Shimizu Corporation, 3-4-17, Etchujima, Koto-ku, Tokyo 135-8530, Japan

ii) Research fellow, Institute of Technology, Shimizu Corporation, 3-4-17, Etchujima, Koto-ku, Tokyo 135-8530, Japan

iii) Senior research engineer, Institute of Technology, Shimizu Corporation, 3-4-17, Etchujima, Koto-ku, Tokyo 135-8530, Japan

\begin{abstract}
Dynamic centrifugal model tests are carried out to confirm the effect that surface improvement using a high-permeability material has on reducing liquefaction damage to small structures. A gravel layer with a prototype thickness of $0.3-1.2 \mathrm{~m}$ was laid under the model structure. Without improvement, the inclination of the model structure continued throughout the period of liquefaction, yielding a residual inclination of about $1 / 6.5$. In cases with improvement by gravel, residual inclinations were $1 / 300$ or less in all cases, even though most of the ground around the model suffered liquefaction.
\end{abstract}

Keywords: liquefaction, drainage, surface improvement, centrifugal model test

\section{INTRODUCTION}

Ground liquefaction during earthquakes causes a great deal of damage to structures, outdoor facilities and infrastructure, requiring expensive and time-consuming repair work. Typical countermeasures against liquefaction include improving the soil to prevent liquefaction or using a pile foundation. However, these measures are generally expensive and have proven difficult to apply in the case of small structures, such as cubicles for high-voltage power connections, water storage tanks, pipe racks, etc. Although such structures are small, liquefaction damage may present a major obstacle to restarting factory operations.

On the other hand, even if the excess pore water pressure ratio of the ground reaches 1.0 during an earthquake, it is possible to maintain the bearing capacity of the ground in this "post-liquefaction" state ${ }^{4)}$ by drainage, and thereby prevent failure. Based on this idea, the authors have proposed countermeasures to reduce liquefaction damage of small structures by improving the surface to provide drainage ${ }^{5)}$. This paper describes centrifugal model tests that clarify the influence of ground improvement work specifications (permeability, thickness and area of the drainage layer) on the inclination of structures during liquefaction.

\section{OUTLINE OF CENTRIFUGAL MODEL TESTS}

A number of dynamic centrifugal model tests were conducted at an acceleration of 30g. The model used in the tests is shown in Figure 1. A laminar box measuring $800 \mathrm{~mm}$ long, $470 \mathrm{~mm}$ wide and $370 \mathrm{~mm}$ deep was used. Experiments were performed under more severe conditions than experienced in reality so as to confirm the reliability of the improvement measures. A liquefiable layer of thickness $240 \mathrm{~mm}(7.2 \mathrm{~m}$ in prototype scale) at $35 \%$ relative density $\left(\gamma_{\mathrm{sat}}=18 \mathrm{kN} / \mathrm{m}^{3}\right)$ was created using silica No. 7 sand. The permeability coefficient of this soil layer was $5.58 \times 10^{-3} \mathrm{~cm} / \mathrm{s}$. Figure 2 shows the grain size accumulation curves of the silica sands used. The pore fluid was silicone oil of $30 \mathrm{~mm}^{2} / \mathrm{s}$ and specific gravity 1.0 ,

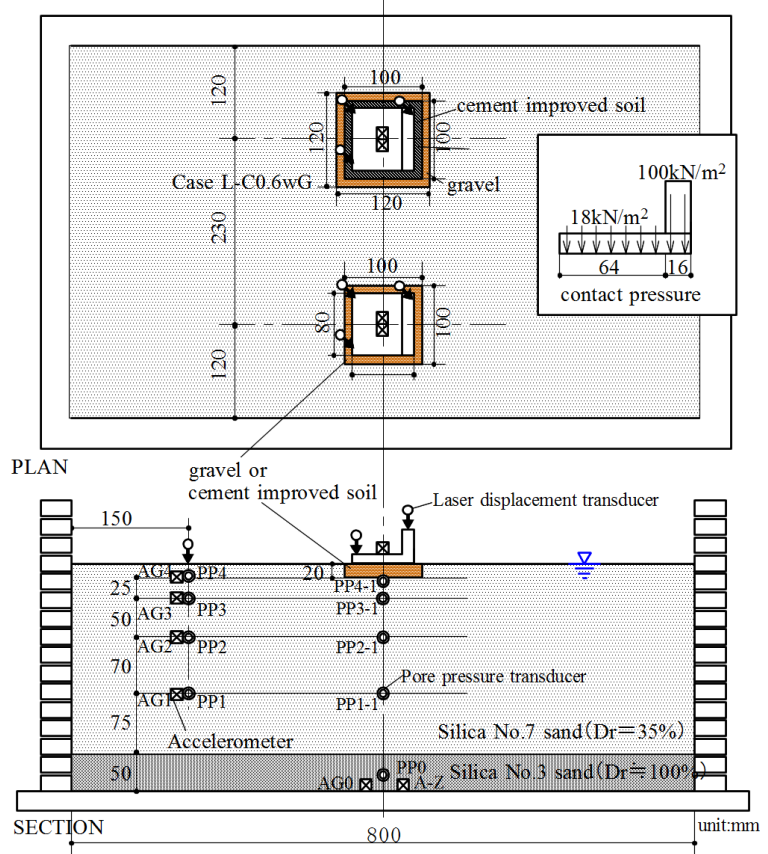

Fig.1 Test model 
and the groundwater level was set at the ground surface. The S-wave velocity of the ground was measured using bender elements as the centrifugal acceleration was increased in the experiment. The relationship between the S-wave velocity of the soil and the effective overburden pressure of the No. 7 sand layer is shown in Figure 3. The $\mathrm{S}$-wave velocity of the soil between the ground surface and $-2 \mathrm{~m}$ (effective pressure about $16 \mathrm{kN} / \mathrm{m}^{2}$ ) was about 50 $-100 \mathrm{~m} / \mathrm{s}$.

The model structure had a square plan with sides of $80 \mathrm{~mm}$ (representing $2.4 \mathrm{~m}$ at prototype scale) and it was weighted eccentrically to ensure that inclination occurred readily, as shown in Figure 1. The eccentric weight consisted of a brass block measuring $16 \mathrm{~mm}$ in width ( $20 \%$ of the foundation width) attached to one edge of the foundation. The ground contact pressure under centrifugal acceleration of $30 \mathrm{~g}$ was about $100 \mathrm{kN} / \mathrm{m}^{2}$ under the brass block, and about $18 \mathrm{kN} / \mathrm{m}^{2}$ elsewhere. The average ground contact pressure was $34 \mathrm{kN} / \mathrm{m}^{2}$. To increase the frictional force on the bottom of the model structure, No.7 silica sand was bonded to it. The two structures were placed into a soil container to be able to compare the results of different conditions.

Table 1 shows the test cases. The experiments involved changing the thickness, area and permeability of the soil improvement material. In addition, a case with dry sand of the same density in place of the liquefiable layer was also carried out for comparison under conditions where liquefaction did not take place. To simulate soil improvement with high permeability, silica No. 3 sand (referred to as gravel) with a relative density of more than $90 \%$ was used. The permeability coefficient of this gravel was $2.9 \mathrm{~cm} / \mathrm{s}$. For soil improvement using a material with no permeability, cement-improved soil was used as the improvement material. This cement-improved soil was prepared to a predetermined shape by adding cement at $80 \mathrm{~kg} / \mathrm{m}^{3}$ to silica No. 7 sand. In case L-C0.6wG, silica No. 3 sand is laid to a width of $0.3 \mathrm{~m}$ and thickness of $0.6 \mathrm{~m}$ around the cement-improved soil of width $3 \mathrm{~m}$ and thickness $0.6 \mathrm{~m}$. This case was designed to confirm the effect of drainage from around the foundation only.

Because the soil is very loose, with a $35 \%$ relative density, the centrifugal loading might itself induce inclination of the structure. In order to prevent this, centrifugal acceleration of $30 \mathrm{G}$ was applied with a counterweight to account for the eccentric load prior to the experiment. The centrifugal acceleration was halted once, and the counterweight was removed. After applying the centrifugal acceleration of $30 \mathrm{G}$ again, the shaking table tests were carried out. Therefore, at the time of actual testing, the soil below the foundation had been overconsolidated.

A $2 \mathrm{~Hz}$ sine wave representing a maximum prototype acceleration of $300 \mathrm{~cm} / \mathrm{s}^{2}$ was used as the input motion. Figure 4 shows the input seismic waveform.

Measurements were taken of structure and ground accelerations, pore water pressure in the ground and settlement of the structures and the ground surface. Structure settlement was measured at three positions for each structure using laser displacement gauges to determine inclination.

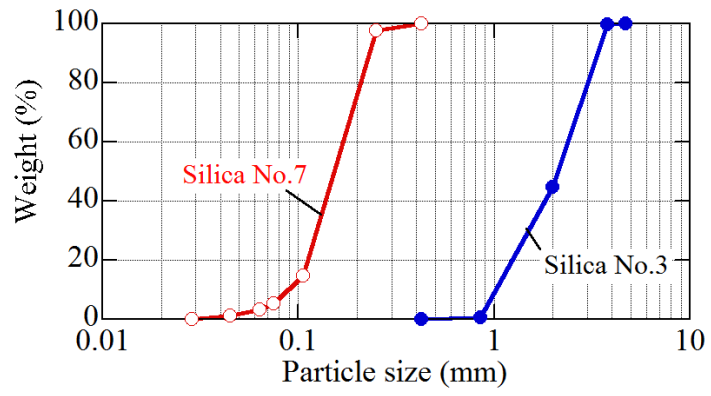

Fig.2 Grain size accumulation curves of silica sand

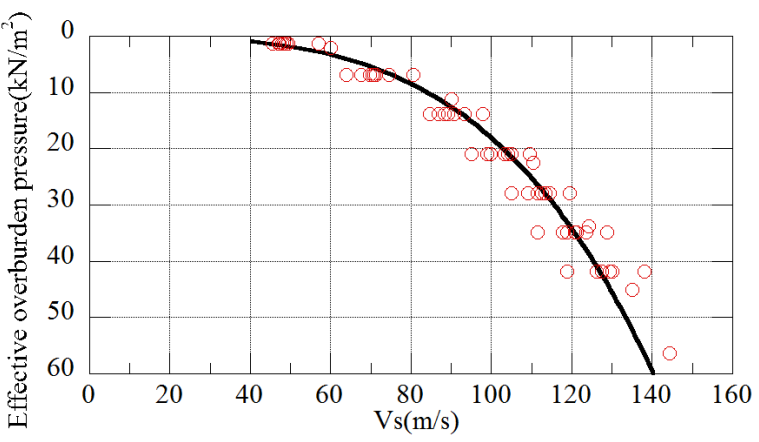

Fig.3 Vertical distribution of S-wave velocity

\begin{tabular}{|c|c|c|c|c|}
\hline \multirow[b]{2}{*}{ Test ID } & \multirow[b]{2}{*}{ Soil } & \multicolumn{3}{|c|}{ Improvement } \\
\hline & & Material & $\begin{array}{l}\text { Thickness } \\
\text { (m) }\end{array}$ & $\begin{array}{c}\text { Width } \\
(\mathrm{m})\end{array}$ \\
\hline L-N & \multirow{10}{*}{$\begin{array}{l}\text { Saturated } \\
\text { sand }\end{array}$} & - & - & - \\
\hline L-G0.3 & & \multirow{5}{*}{$\begin{array}{l}\text { Gravel } \\
\text { (Silica } \\
\text { No.3) }\end{array}$} & 0.3 & \multirow{3}{*}{3.0} \\
\hline L-G0.6 & & & 0.6 & \\
\hline L-G1.2 & & & 1.2 & \\
\hline L-G0.3-3.6 & & & 0.3 & 3.6 \\
\hline L-G0.3-4.8 & & & 0.3 & 4.8 \\
\hline L-C0.3 & & \multirow{3}{*}{$\begin{array}{c}\text { Soil } \\
\text { cement }\end{array}$} & 0.3 & \multirow{3}{*}{3.0} \\
\hline L-C0.6 & & & 0.6 & \\
\hline L-C1.2 & & & 1.2 & \\
\hline L-C0.6wG & & $\begin{array}{c}\text { Soil } \\
\text { cement }+ \\
\text { Gravel }\end{array}$ & 0.6 & $3.0+0.6$ \\
\hline D-N & Dry sand & - & - & - \\
\hline
\end{tabular}

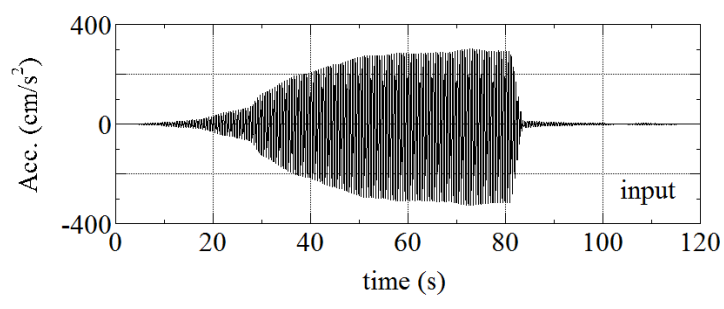

Fig.4 Input wave 

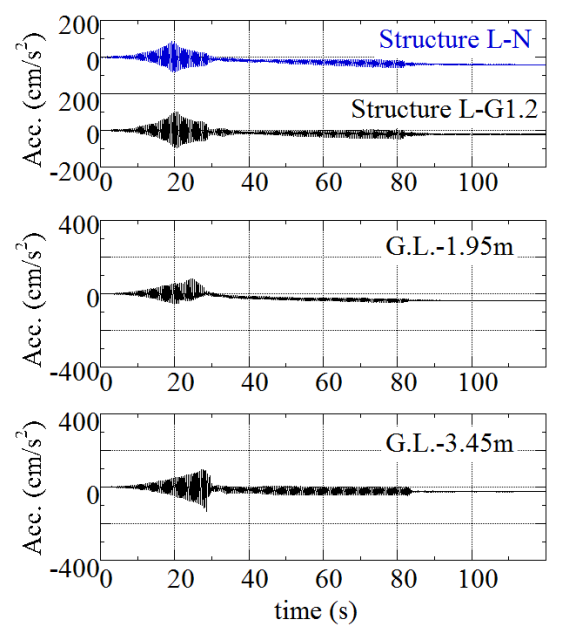



Fig. 5 Time histories of acceleration, pore water pressure and excess pore water pressure ratio

\section{TEST RESULTS}

Test results are given in prototype scale.

\subsection{Effects of surface improvement by gravel}

Figure 5 shows the time histories of acceleration, soil pore water pressure and soil excess pore water pressure for case L-N (without ground improvement) and case L-G1.2, which both involved the same ground. The excess pore water pressure ratio (EPWPR) of the soil just below the structures was determined using the sum of stress increment due to the weight of the structure under its center and the overburden pressure of the soil.

Around the sides, EPWPR of the ground reached 1 in about 20-30 seconds after the start of shaking. The Silica No. 7 sand layer was determined to have reached the liquefaction state in all layers. Acceleration of the structures began to decrease at 20 seconds, and by about 30 seconds the acceleration was very small. No differences in structure acceleration relating to ground improvement were observed. Although the pore water pressure of the soil after 20-30 seconds at GL- $1.2 \mathrm{~m}$ appears to be highly variable, this is thought to result from some kind of noise because the same phenomenon was not observed in other cases. In case L-N, the time history of pore water pressure at GL- $1.2 \mathrm{~m}$ under the structure foundation was almost the same value as in the ground to the side of the structure. On the other hand, where the ground had been improved with a $1.2 \mathrm{~m}$ thickness of gravel (L-G1.2), the increment of pore water pressure at the same depth was slight. However, at GL-1.95m and $-3.45 \mathrm{~m}$, the time history of pore water pressure was substantially the same as for case L-N, so the effect of improvement using gravel was small. In both cases, the excess pore water pressure ratio just under the structure did not reach 1 because of the weight of the structure. However, the acceleration time histories of the structure and the liquefied ground were similar, and there was a plateau in the time history of pore water pressure; this is evidence that the ground under the structure suffered liquefaction.

Figure 6 shows a time history of pore pressure after the end of shaking. Because of the non-draining condition at
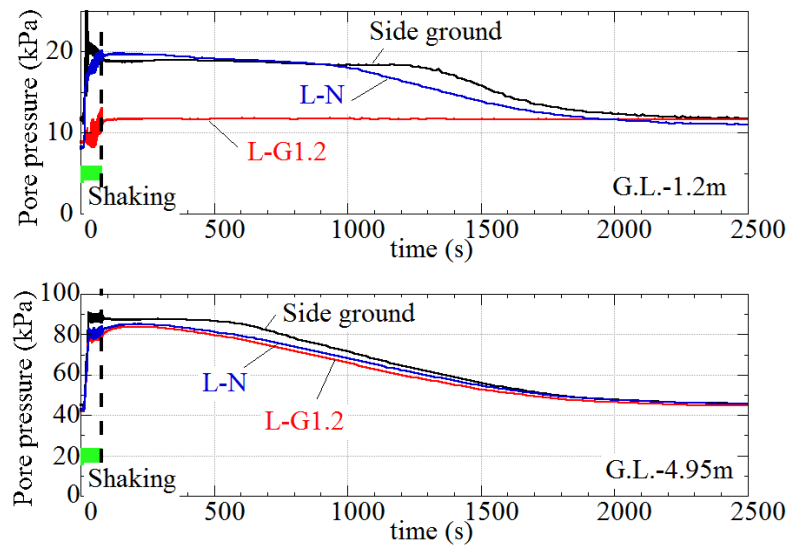

Fig.6 Time histories of pore water pressure



Fig.7 Time histories of average settlement of structure

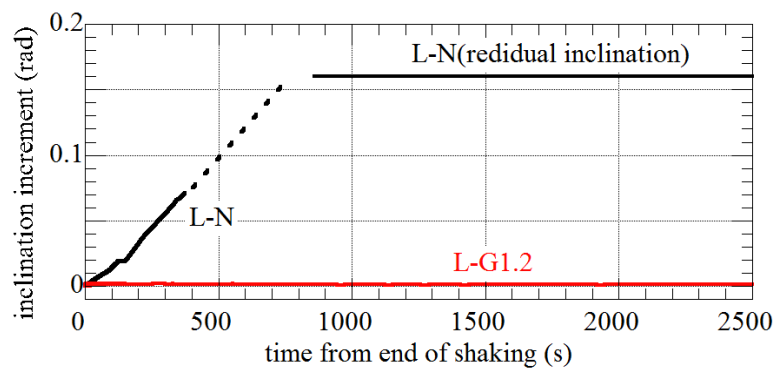

Fig.8 Time histories of inclination change of the structure after shaking stopped 
the base of the soil container, the dissipation of pore water pressure started from the deep in the ground. In this case, the liquefied state continued for about 500 seconds at GL-4.95m and about 1200 seconds (about 20 minutes) at GL- $1.2 \mathrm{~m}$ to the side of the structure.

Comparisons of the average settlement and inclination change of the structure after shaking stopped are given in Figures 7 and 8. The displacement sensor on the opposite side of the eccentric load became impossible to measure at about 400 seconds due to excessive inclination in case L-N (without countermeasures). Settlement of the structure increased rapidly during shaking and then continued slowly after shaking stopped. Although the settlement in case L-N was slightly greater, there was no significant difference in settlement according to whether improvement measures were in place. In contrast, significant differences were observed in the inclination of the structure after end of the shaking. In the case with improvement measures (L-G1.2), the inclination of the structure hardly increased. In the case without measures $(\mathrm{L}-\mathrm{N})$, the inclination of the structure continued to increase after shaking stopped, and the final inclination increment after shaking was about 0.16 (about 1/6.3) in measurements after the test.

Changes in inclination after shaking stopped in the cases with drainage improvement are shown collectively in Figures 9 and 10. Inclination ended quickly in all cases. In spite of the very severe experimental conditions, the final inclination was within about $1 / 300$ or less. In the range of the experimental conditions, the thickness and area of the gravel layer had little effect on the final inclination of the structure. It is confirmed that, by ensuring drainage, a gravel layer with a thickness of about $0.3 \mathrm{~m}$ limits the inclination of a structure.

\subsection{Influence of the permeability of improvement}

The time histories of soil pore water pressure with and without drainage provided by ground improvement are compared in Figure 11 for cases where the improvement thickness is $0.6 \mathrm{~m}$. In the case of soil cement improvement, the time histories of pore water pressure just below the improved section are almost the same as in the ground to the side. At G.L. $-1.35 \mathrm{~m}$, in the case of soil cement improvement (L-C0.6), the excess pore water pressure continued increasing slightly after the end of shaking, while in the case of gravel improvement (L-G0.6) this increase in pore water pressure was halted.

Figure 12 shows time histories of the dissipation of pore water pressure at GL-1.35m. It took 900-1000 seconds after shaking stopped before the pore water pressure began to fall.

The time histories of inclination after shaking stopped are shown in Figure 13. Here, since the improvements covered an area $0.6 \mathrm{~m}$ greater than that of the structure foundation, the apparent size of the foundation was greater. The increases in inclination in the cases of soil cement improvement are less than that in the case without improvement measures (L-N). But in cases with soil cement improvement, the inclination of the structure continued to increase after shaking stopped, up to about 900 seconds when the pore water pressure near the ground

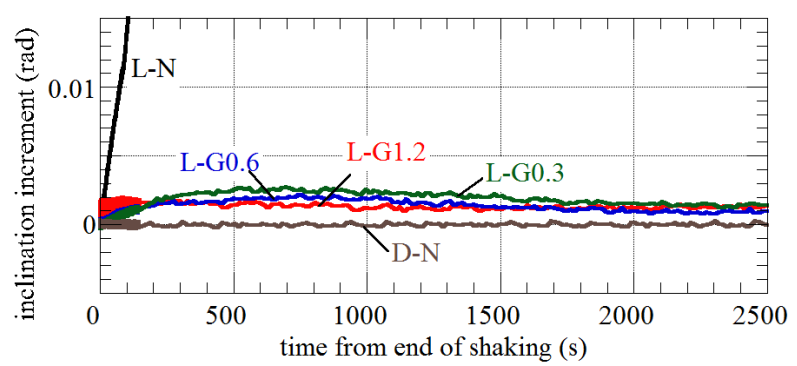

Fig.9 Time histories of inclination change of the structure after shaking stopped (Influence of improvement thickness)

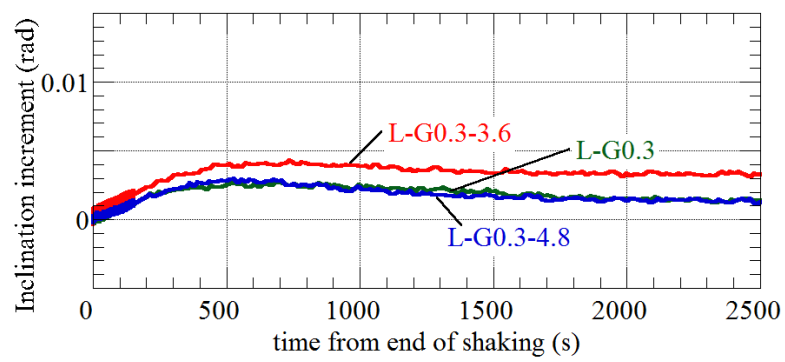

Fig.10 Time histories of inclination change of the structure after shaking stopped (Influence of improvement area)
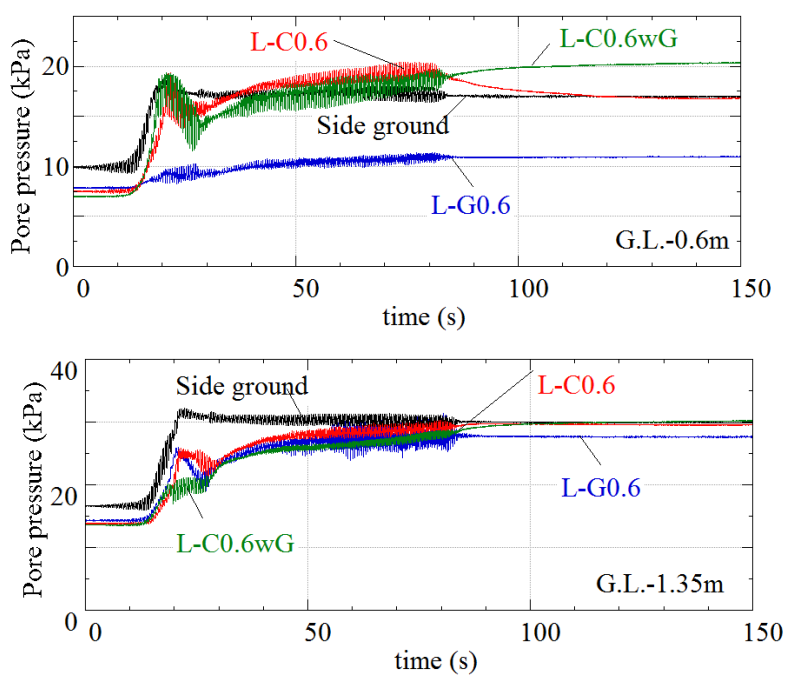

Fig.11 Time histories of pore water pressure (Influence of permeability of improvement)

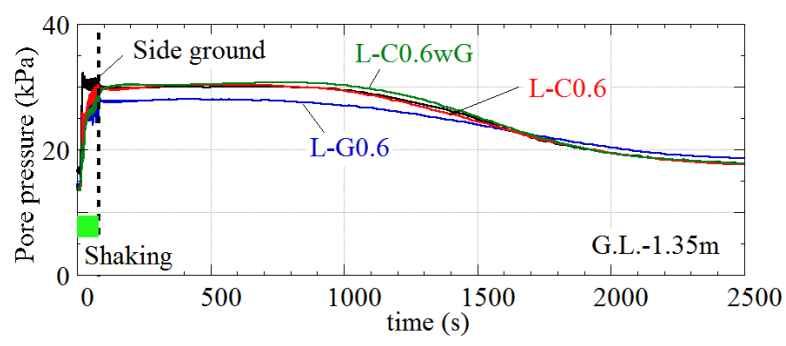

Fig.12 Time histories of pore water pressure (Influence of permeability of improvement) 
surface began to decrease. The final inclination increment after shaking was about $0.065(1 / 150)$ to $0.01(1 / 100)$. In cases with soil cement improvement, the depth of the ground improvement has little inclination-limiting effect.

The structure continued to incline further until the pore water pressure began to decrease, so it can be said that if liquefaction is prolonged, as in real ground, improvement using soil cement leads to a risk of very large inclinations developing. The inclination increment in case $\mathrm{L}-\mathrm{C} 0.6 \mathrm{wG}$, where gravel was laid around the soil cement improved area, was almost the same as in case L-C0.6. So it can be said that improving drainage only around the foundation has little effect on reducing the inclination of the structure. The condition of the test structures at the end of the test is

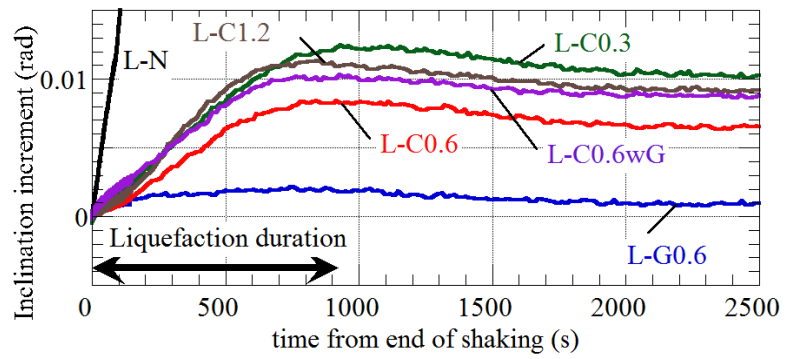

Fig.13 Time histories of inclination change of the structure after shaking stopped (Influence of permeability of improvement)



Photo 1 Status of the test structures after test (L-G1.2 and L-N)

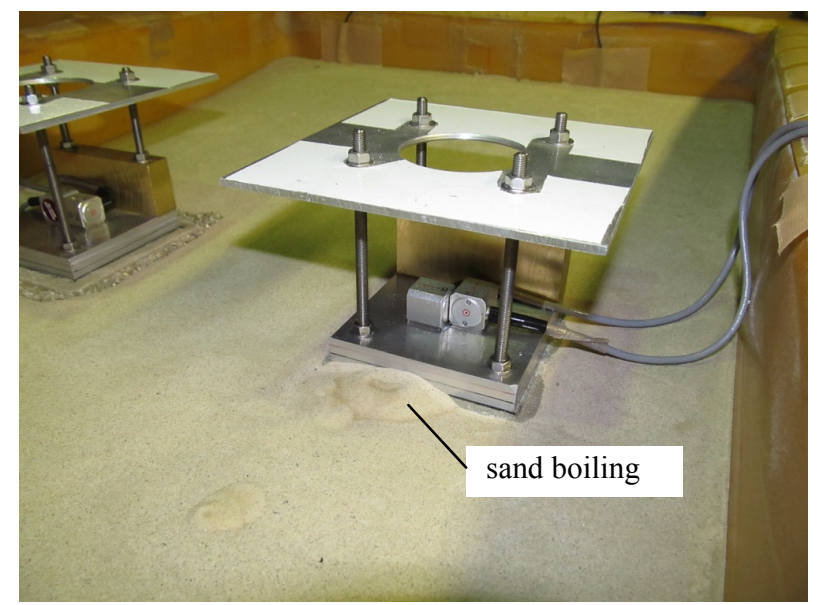

Photo 2 Status of the test structures after test (L-C1.2) shown in Photo 1 and 2.

In the case without improvement measures (L-N), the structure has inclined significantly, but with gravel improvement (L-G1.2), the inclination is small and no sand boiling is observed near the structure. In cases with improvement by soil cement (L-C1.2), sand boiling occurred around the improved area.

\section{CONCLUSION}

Through centrifugal tests using a model structure, it was confirmed that liquefaction damage can be reduced by placing a gravel layer under a structure. Although the conditions set in the tests were very severe, the final inclination of the model structure after shaking had stopped was very small. This confirms that structural damage due to liquefaction can be reduced significantly by applying improvement measures near the ground surface. In cases where improvement was carried out using soil cement with no permeability, the inclination of the structure continued to increase throughout the period of soil liquefaction. This demonstrates the importance of ensuring good drainage in reducing liquefaction damage by surface improvement.

\section{REFERENCES}

1) Yasuda, S., Ishihara, K., Harada, K. and Shinagawa, N. (1996): Effect of soil improvement on ground subsidence due to liquefaction, Special Issue of Soils and Foundations, 99-107

2) Sasaki, Y. and Taniguchi, E. (1982): Shaking table tests on gravel drains to prevent liquefaction of sand deposits, Soils and Foundations, 22(3), 1-14

3) Suzuki, Y., Saitoh, S., Onimaru, S., Kimura, T., Uchida, A. and Okumura, R. (1996): Grid-shaped stabilized ground improved by deep cement mixing method against liquefaction for building foundation, Tsuchi to Kiso, 44(3), 46-48 (in Japanese)

4) Shamoto, Y., Zhang, J. and Tokimatsu, K. (1998): New charts for predicting large residual post-liquefaction ground deformation, Soil Dynamics and Earthquake Engineering, 17, 427-438

5) Shamoto, Y., Kimura, T., Mano, H., Yoshinari, K. and Ishikawa, A. (2012): Liquefaction damage reduction effect of small-scale structure by surface improvement (part 1-5), 47th Japan National Conference on Geotechnical Engineering, 1383-1392 (in Japanese) 